and the end of observation. And ECG parameters (QT, OTC, OTd, Tp-e, Tp-ed, Tp-e/OT) were assessed all through the duration.

Results PES results showed that DPT, VERP and VFT significantly decreased in HCN4 group compared to vehicle group. At the same time, ECG results displayed that QTc, QTd, Tp-e, Tp-ed, Tp-e/QT all increased after HCN4-MSCs injection.

Conclusion In an CAVB model, MSCs loaded with HCN4 transplantation may induce electrical ventricular instability. Although biological pacemaker may have promising future using HCN gene delivery and cell implantation, we suggest that such preclinical evaluation should be carefully carried out.

\section{[gw22-e0905] HCN4 MODIFIED MESENCHYMAL STEM CELLS INJECTION AFFECTS VENTRICULAR ELECTROPHYSIOLOGY IN COMPLETE ATRIOVENTRICULAR BLOCK CANINE}

Lu Wei Southwest Hospital

\subsection{6/heartjnl-2011-300867.246}

Background HCN family of pacemaker channel genes has been investigated to create biological pacemaker through cell transplantation using viral vectors or adult human mesenchymal stem cells (hMSC) as delivery platforms. A potential concern about biological pacemakers is the possibility that their malfunction may induce some kind of arrhythmia. We investigated cardiac electrical stability after pacemaker cell transplantation to the epicardium myocardium.

Methods we used lentivirus vector to integrate the mHCN4 gene into the genome of dMSCs and were able to achieve stable expression of the mHCN4 channel gene permanently. Intact dogs were randomised into two groups: a vehicle group injected with LV-EGFP-dMSCs into the myocardium, and a HCN4 group injected with LV-EGFP-HCN4-dMSCs. After injection for seven days, each dog was interventioned into complete atrioventricular block (CAVB) model by radiofrequency ablation of the His bundle and cardiac pacemaker was implanted as tandem model. Spontaneous rhythms can be recorded through Electrocardiogram observation for 14-28 days. Programmed Electrical Stimulation (PES) was performed to evaluate further a cardiac substrate for arrhythmia susceptibility. Cardiac electrophysiological parameters, including diastolic pacing threshold (DPT), ventricular effective refractory period (VERP), VFT were determined before the injection 